Protocol

\title{
Honeybee Cognition as a Tool for Scientific Engagement
}

\author{
Jai A. Denton ${ }^{1,2}$, Ivan Koludarov ${ }^{3}$, Michele Thompson ${ }^{4}$, Jarosław Bryk ${ }^{5}$ (D) and Mariana Velasque ${ }^{1, *}$ \\ 1 Genomics \& Regulatory Systems Unit, Okinawa Institute of Science \& Technology, 1919-1 Tancha, \\ Kunigami-gun 904-0495, Japan; jai.denton@monash.edu \\ 2 Institute of Vector-Borne Disease, Monash University, Clayton, VIC 3800, Australia \\ 3 Animal Venomics Group, Justus Liebig University, 35390 Giessen, Germany; \\ Ivan.Koludarov@agrar.uni-giessen.de \\ 4 Independent Researcher, Dexter, MI 48130, USA; michelez@umich.edu \\ 5 School of Applied Sciences, University of Huddersfield, Huddersfield HD1 3DR, UK; j.bryk@hud.ac.uk \\ * Correspondence: mariana.velasque@oist.jp
}

Citation: Denton, J.A.; Koludarov, I.; Thompson, M.; Bryk, J.; Velasque, M. Honeybee Cognition as a Tool for Scientific Engagement. Insects 2021, 12, 842. https://doi.org/10.3390/ insects 12090842

Academic Editors: Cristina

Castracani and Alessandro

Campanaro

Received: 2 August 2021

Accepted: 14 September 2021

Published: 18 September 2021

Publisher's Note: MDPI stays neutral with regard to jurisdictional claims in published maps and institutional affiliations.

Copyright: (c) 2021 by the authors. Licensee MDPI, Basel, Switzerland. This article is an open access article distributed under the terms and conditions of the Creative Commons Attribution (CC BY) license (https:// creativecommons.org/licenses/by/ $4.0 /)$.
Simple Summary: Global scientific literacy can be improved through widespread and effective community engagement by researchers. We propose honeybees (Apis mellifera) as a public engagement tool due to widespread awareness of colony collapse and the bees' importance in food production. Moreover, their cognitive abilities make for engaging experiments. Their relative ease of cultivation means that studies can be performed cost-effectively, especially when partnering with local apiarists. Using a proxy for honeybee learning, a group of non-specialist high-school-aged participants obtained data suggesting that caffeine, but not dopamine, improved learning. This hands-on experience facilitated student understanding of the scientific method, factors that shape learning and the importance of learning for colony health.

Abstract: Apis mellifera (honeybees) are a well-established model for the study of learning and cognition. A robust conditioning protocol, the olfactory conditioning of the proboscis extension response (PER), provides a powerful but straightforward method to examine the impact of varying stimuli on learning performance. Herein, we provide a protocol that leverages PER for classroombased community or student engagement. Specifically, we detail how a class of high school students, as part of the Ryukyu Girls Outreach Program, examined the effects of caffeine and dopamine on learning performance in honeybees. Using a modified version of the PER conditioning protocol, they demonstrated that caffeine, but not dopamine, significantly reduced the number of trials required for a successful conditioning response. In addition to providing an engaging and educational scientific activity, it could be employed, with careful oversight, to garner considerable reliable data examining the effects of varying stimuli on honeybee learning.

Keywords: citizen science; honeybee learning; memory; proboscis extension response; associative learning; honeybee cognition

\section{Introduction}

We currently face an unprecedented combination of events, ranging from pandemics and a climate emergency to desertification of arable lands and rapid decline in biodiversity, challenges whose understanding and overcoming demands broad scientific expertise. Thus, it is imperative that communities not only understand the scientific method, but also engage with it [1-3]. A key approach to drive this engagement is through citizen science activities. Although there is not a single concise definition of citizen science, it can typically be thought of as involving non-scientists in the scientific process. However, many of the most significant programs either engage with community members already scientifically literate or are much more goal-focused and thus do not necessarily improve understanding of the scientific method [4-6]. Therefore, effective community engagement and citizen 
science need to include both tentpole and smaller activities with community members that are underrepresented in the scientific process.

Although citizen science and scientific outreach take many forms, it is most effective when participants can meaningfully engage with the topic $[7,8]$. The central activity, experiment or scientific question needs to incite enthusiasm but must also be presented in a framework that ensures participants gain the most from the experience [7,8]. Ultimately, citizen science programs are not only beneficial for students, but also help to build positive relationships between universities that support them and the local community. Due to their broad appeal, external funding from private donors and corporate grants, with more flexible spending parameters, is often available and can be easier to secure.

The Ryukyu Girls scientific engagement program seeks to engage female high school students, around 15 years old, from the Japanese Okinawa prefecture in the scientific process. This voluntary program, run over a weekend, included, in addition to the activity described herein, several talks by prominent and upcoming scientists. As part of this program, we developed an experiment that sought to teach the scientific method as a way of interacting with the world. We employed clear and simple language when describing the scientific processes to ensure it was accessible to non-native English speakers. The program was taught in two languages, English and Japanese, with simultaneous translation provided. Moreover, the presenter shared her experiences as a young female scientist and thereby sought to not only a connection with participants but to demonstrate a pathway for young women in science. As a result, we hope participants were deeply engaged with the content, but also developed a level of trust with the presenter.

In addition, a critical component of our approach was in highlighting the importance of participation in the scientific process. By drawing a connection between their participation as a part of a larger effort to increase underrepresented voices in science, participants became emotionally invested and, as a result, experienced meaningful engagement in the experiment.

Insects are an essential part of most, if not all, ecosystems [9-12]. They provide multiple ecological functions, ranging from breaking down organic matter in the soil to pollination and the control of insect and plant pests [13-15]. Their diversity and abundance is directly related to the state of conservation of the environment, with more natural and undisturbed areas having a higher diversity and abundance than disturbed areas [16-19].

Apis mellifera (European honeybees) provides an excellent tool for scientific engagement, of academic and lay public alike, while furthering our understanding of this critical agricultural pollinator. Honeybees have complex social interactions driven by intra-hive learning and communication [20]. They are capable of not only learning the location of food resources but also communicating to their nestmates through varying forms of communication including the waggle dance $[20,21]$. In the laboratory, honeybees are exceptional model organisms to study cognition, memory and communication. Honeybees are capable of complex cognitive processes. For instance, honeybees can memorise locations, patterns, faces and even understand conceptual relationships, such as above/below and same/different $[22,23]$. Despite this great potential for honeybees as models in cognitive neuroscience, their use remains limited compared to Drosophila melanogaster [24].

Classical conditioning is a form of conditioning on which a subject learns to associate a neutral stimulus, called conditioned stimulus (CS), with a stimulus of biological significance, the unconditioned stimulus (US), such as sucrose [25]. Over time, animals start to associate the initial neutral stimulus to the US, acquiring the capacity to elicit a conditioned response. Although classical conditioning is considered to be a basic learning process, it has become the foundation of cognition and memory studies in animals, especially in insects [26-31]. Among insects, honeybees are considered one of the most robust organisms for the study of classical conditioning $[32,33]$. Such success is mainly due to the presence of several powerful conditioning protocols [29,30,34-36].

Honeybees extend their proboscis when their chemoreceptors enter into contact with sucrose. When sucrose is paired with another stimulus, such as a distinctive scent or a 
visual pattern, honeybees can learn to anticipate the sugar reward when exposed to the stimulus. This results in an easily observed anticipatory phenotype of proboscis extension. In conditioning, a naive bee (i.e., a honeybee without any previous experience to the stimulus) is exposed to a neutral stimulus (i.e., a scent or an image it has not being exposed to before), CS, followed by a sucrose reward, US [29,30,34]. During conditioning, the honeybee learns to associate the initially neutral stimulus, CS, to the US (i.e., sucrose). Although honeybees are amenable to cognitive study, and are cost-effective and ubiquitous worldwide, conditioning protocols rely on the implementation of several procedural steps [37]. Such steps, such as training trials, can be laborious and require large sample sizes, which can be an impediment to obtaining statistically robust findings [38]. Therefore, the development of systems that provides large-scale data in a semi-structured system, such as citizen science activities and/or automation, can help to overcome these challenges.

Herein we describe the use of $A$. mellifera learning as an experimental system by investigating how dopamine and caffeine affects learning performance in honeybees. The study was implemented by the Ryukyu Girls engagement program, as an example of outreach and citizen science activities. The results indicated that caffeine-treated bees learn faster than dopamine-treated and control bees. All data were collected by the participants of the programme and analysed as a classroom-based demonstrator-led group activity. There was little data loss, and results were comparable to the pilot trial, which suggests that the protocol, and programmes such as Ryukyu Girls in particular, can be used to support large-scale observation and data collection.

\section{Methods and Protocol}

\subsection{Honeybee Sourcing and Handling}

All bees used in the experiment were from a single hive. Newly emerged bees were obtained by removing two frames containing capped larvae from the hive, brushing off adult bees and placing the frames in a small hive box for $6 \mathrm{~h}$. To maintain optimal conditions for honeybees, small hives were kept inside the incubator, with constant temperature of $34{ }^{\circ} \mathrm{C}$ and $60 \%$ humidity. After $6 \mathrm{~h}$, all emerged honeybees were collected and harnessed for experimentation. The two frames were returned to the hive as soon as possible after removing the newly emerged bees.

\subsection{Harnessing}

After being anesthetized using ice [39], bees were harnessed using plastic drinking straws, approximately $14 \mathrm{~mm}$ in diameter, and cut to lengths of approximately $2 \mathrm{~cm}$ with a diagonal section removed from one end, creating a $V$, to allow the bee's head to protrude as described by [40]. Anesthetized bees were placed inside the straw piece, restricting the movement of their legs. The head was fixed in place with small pieces of masking tape that allowed movement of the antennae and proboscis. Although this process is well described by Scheiner et al. [37], harnessing requires patience and practice to effectively perform. Harnessed bees were divided into three groups, providing three bees per treatment group to the students. To facilitate handling and reduce confusion associated with the treatment, harnessed bees were placed in small holes present in a Styrofoam tray, containing a colour coded indication and names of the treatment groups. To reduce mortality and stress, bees were harnessed $6 \mathrm{~h}$ prior to the practical by the authors without the support of the Ryukyu Girls class.

\subsection{Quantifying Learning}

Despite honeybees being a well-established model organism, there is mixed evidence as to whether newly emerged bees are able to show learning [41]. Therefore, a pilot experiment was conducted to ensure newly emerged honeybees would be capable of associative learning.

Learning performance was measured as the number of trials required for the honeybee to learn a new stimulus. In this case, bees were exposed to a lemon scent while offering 
the sugar solution (sucrose solution supplemented with a scent). When offered a sugar syrup reward, bees extend their proboscis. Over a few trials (i.e., odour paired with sugar reward) the bee learns to associate the odour to the reward, extending their proboscis when exposed to the odour alone. Fewer trials indicates higher learning performance.

\subsection{Pilot Study}

Pilot was performed in 18 bees ( 6 bees per treatment group) and made it possible to establish the presence of associative learning in newly emerged honeybees and if there was a clear trend according to the treatment group.

\subsection{Pre-Experimental Preparation}

Because dopamine and caffeine differ with respect to their assimilation and reabsorption time, depending on the organism and age, all bees were fed three times prior to the experiment. Feeding was done at 2,1 and $0.5 \mathrm{~h}$ before the experiment with a sucrose solution mixed with the proposed treatment. Bees were fed with sucrose solutions $(50 \% w / w)$ without additives (control), with $0.001 \%$ caffeine (caffeine supplement, Allmax Nutrition, Carson City, NV, USA) or with $0.001 \%$ dopamine (Dopamine Plus N-care, Japan). No odours were presented during this feeding.

The $50 \%(w / w)$ sucrose solution was prepared by mixing $200 \mathrm{~mL}$ reverse osmosis purified water with $100 \mathrm{~g}$ of sucrose and stirred until all sugar was dissolved. The sucrose solution was divided in three equal parts. One part was mixed with $1 \mathrm{mg}$ of caffeine and another with $1 \mathrm{mg}$ of dopamine. Both solutions were stirred until the chemical was dissolved. All solutions were allocated in $1 \mathrm{~mL}$ Eppendorf tubes to be used during the practice.

To create the odour stimulus during the trials, one drop of lemon essential oil (Lemon Essential Oil; My Pure Earth, Japan) in a cotton ball was used. To prevent evaporation and further contamination, the cotton ball was stored inside an Eppendorf tube. All solutions used during the pre-preparation stage and during experimental training were prepared $18 \mathrm{~h}$ prior to the practice. All material used in the experimental training was labelled and colour coded, following the same Styrofoam tray scheme.

\subsection{Experimental Training}

Prior to the experiment, all Ryukyu Girls participants were provided with an explanation of the motivation, literature review (i.e., effects of dopamine in humans and other animals) and what a hypothesis is. Subsequently, they were shown the experimental setup and divided in twelve groups of three. Each group received 3 copies of the "laboratory notebook" (see Supplementary File S1 for a copy of the laboratory notebook), Eppendorf tubes containing the treatment solutions, the odour scent and multiple swabs. They were then asked to formulate a hypothesis and a prediction for the experiment. They were informed about the risks related to honeybees and how to proceed in case bees escape their harness.

The training trial consisted of approximating the Eppendorf tube containing the lemon scent near the honeybee antennae followed by lightly touching the antennae with the sucrose cotton swab until the bee extends its proboscis, followed by allowing bees to feed for one second (Figure 1). The sucrose cotton swab contained either dopamine, caffeine or just sucrose solution, according to the bee experimental group. Each trial was then repeated across all groups. They were instructed to repeat the trial multiple times. At the third training trial, students were instructed to delay the sucrose reward for a few seconds to allow visualization of the associative learning. Such small delay would allow students to visualise proboscis extension without impairing further training protocol. Therefore, learning would be accounted for only after the third training trial. 


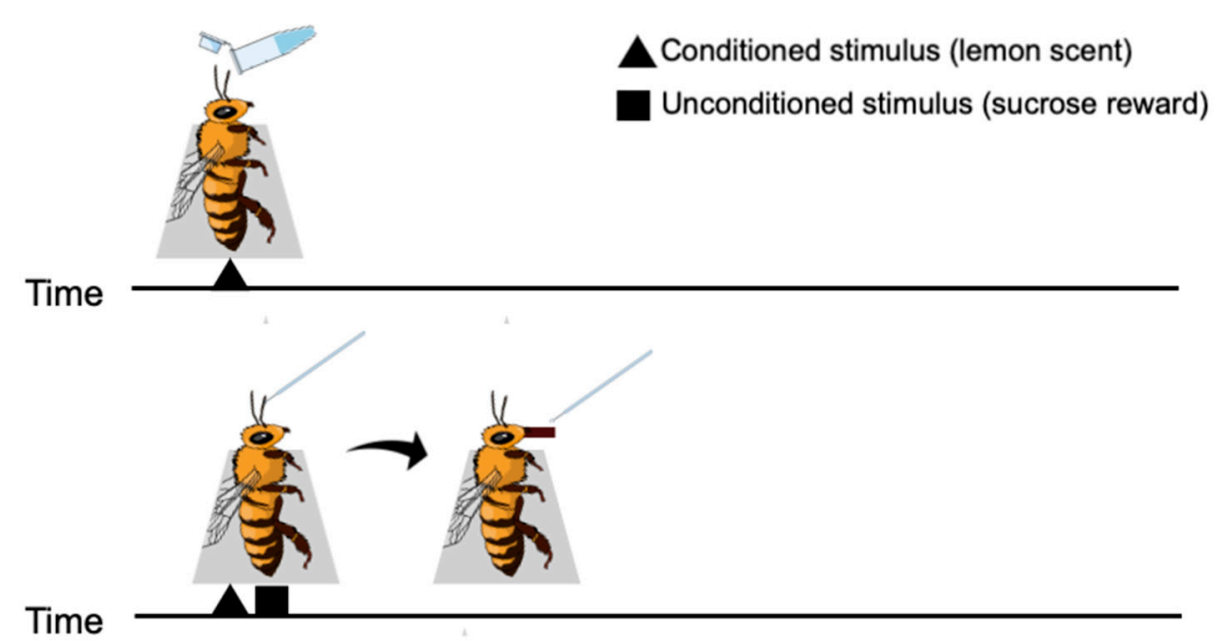

Time

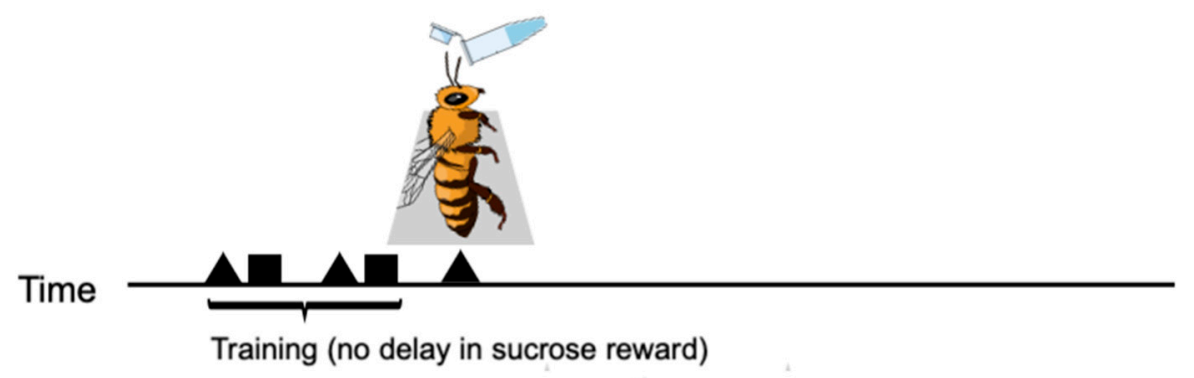

Time

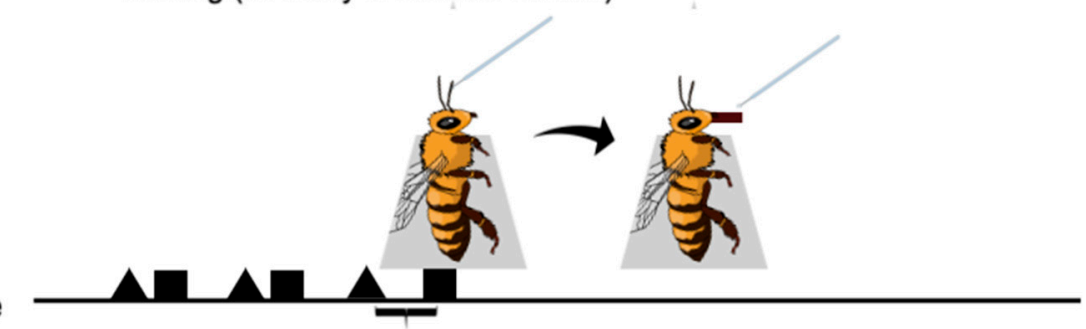

Delay sucrose reward a few seconds

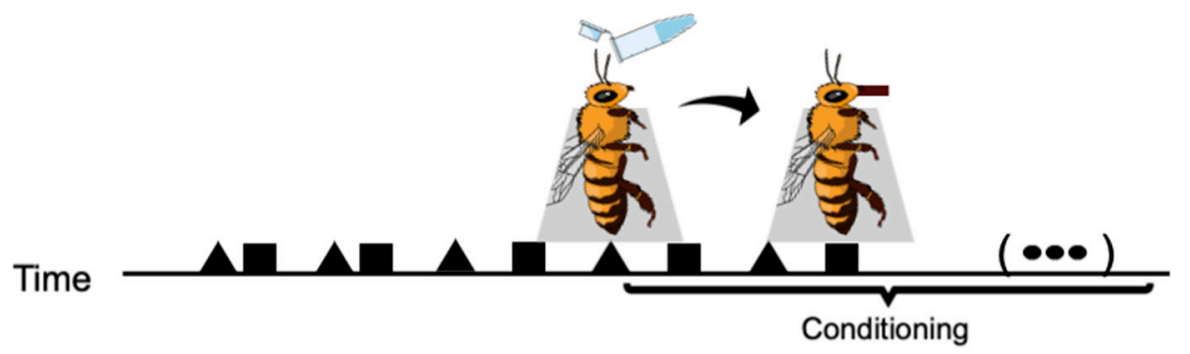

Figure 1. The training schematic for the conditioning of honeybees using a lemon scent. The number of conditioned/unconditioned stimuli cycles can be varied. The conditioning was repeated across all treatments.

We considered that the bee learned when they exposed their proboscis to the lemon scent prior to the offer of the sucrose solution. The number of trials per bee per group was recorded in the lab notebook and supplied to the authors for posterior analysis.

\subsection{Cost}

Around $\$ 100$ (USD) for the supplied hive. The use of newly emerged bees for the practice did not cause significant damage to the hive and it could be used in further experiments. 


\subsection{Student Group and Engagement}

To facilitate learning and increase student engagement, we developed a PowerPoint presentation and a lab notebook. Both the presentation and lab notebook contained the research background and the protocol for the experiment (lab notebook and presentation can be found in the supplemental materials).

After completing the experimental work, data collation and a brief analysis was conducted within a classroom setting. This was closely linked to the aforementioned hypothesis generation, and thus provided participants with a further understanding of hypothesis testing.

\subsection{Risk Management}

When employing an experimental system that presents the possibility of stinging and subsequent allergy risk, diligence is required. We employed several strategies to ensure no participants were stung. Although we conducted an initial survey of participants regarding their allergy status to honeybees, this is insufficient to minimise risk. Where possible, double containment was employed. We used only European newly emerged bees, less than $20 \mathrm{~h}$ old, as they are both less aggressive and do not produce venom [42]. We also developed a harness-like system (see Harnessing) that prevents contact with the bee abdomen. We ensured staff levels were such that every experimental group could be supervised by a demonstrator so if a bee did escape, it could be quickly caught. As a final precaution we also kept an adrenaline injector (EpiPen) in our medical kit.

\subsection{Data Analysis}

Behaviour is one of the most labile phenotypes, and thus use of an appropriate statistical analysis is required to accommodate experimental variation. To minimise issues related to handling, repeated measures ANOVA was employed to analyse the effect of caffeine and dopamine on learning performance. Repeated measures ANOVA compares the difference between means across the treatment groups that are based on repeated observations. Differences between groups were estimated using Multiple Comparisons of Means (Tukey Contrasts) using Bonferoni correction to account for multiple testing. All data analysis was performed in $\mathrm{R}(\mathrm{R}$ version 4.0.2) and can be viewed at: https:/ / github. $\mathrm{com} / \mathrm{marivelasque/HoneybeeOutreach.git.}$

\section{Results}

\subsection{Hypothesis Development}

To facilitate engagement and understanding, considerable classroom time was dedicated to the process of developing a hypothesis and subsequent testing. Unfortunately, due to time and logistical constraints, hypotheses could not be generated spontaneously within the classroom. However, after describing the context, materials available and providing guidance in the form of classroom discussion, participants developed hypotheses nearly identical to those we sought to test.

\subsection{Data Reliability, Collation and Analysis}

A total of twelve student groups performed each experiment in triplicate. However, due to loss of bees or experimental error, the groups averaged 2.4 bees per treatment. Each experimental treatment comprised, on average, 28.6 data points. Of the total 108 data points collected, 22 failed. This data loss was predominantly related to the harnessing of the bees (see Harnessing). Newly emerged bees have softer cuticles than adults [43], and thus improper handling during harnessing and training could be partially responsible for the high mortality.

\subsection{Group Results}

Caffeine, but not dopamine, was found to significantly reduce the number of trials required for a successful conditioning response (Table 1; Figure 2; Supplementary Table S1). 
Each of the 12 groups conducted the control, caffeine treatment and dopamine treatment, in triplicate, by measuring the number of trials required for a conditioning response. Although the average was lower for both caffeine and dopamine treatments, only caffeine had a statistically significant difference (caffeine $p=0.038$, dopamine $p=0.252$; repeated measures ANOVA).

Table 1. Collated data from the 12 student groups. Values indicate the average number of trials required for conditioning response.

\begin{tabular}{cccc}
\hline Group ID & Dopamine & Control & Caffeine \\
\hline 1 & 6.00 & 5.67 & 4.50 \\
2 & 4.00 & 5.00 & 5.67 \\
3 & 6.33 & 6.00 & 4.00 \\
4 & 5.00 & 7.00 & 5.00 \\
5 & 5.00 & 4.50 & 5.00 \\
6 & 4.00 & 4.00 & 4.50 \\
7 & 5.00 & 6.50 & 5.00 \\
8 & 6.33 & 7.00 & 4.00 \\
9 & 4.67 & 5.00 & 5.67 \\
10 & 6.00 & 6.00 & 6.00 \\
11 & 5.00 & 6.67 & 5.00 \\
12 & 4.00 & 6.33 & 4.67 \\
\hline Average & 5.11 & 5.81 & 4.92 \\
\hline
\end{tabular}

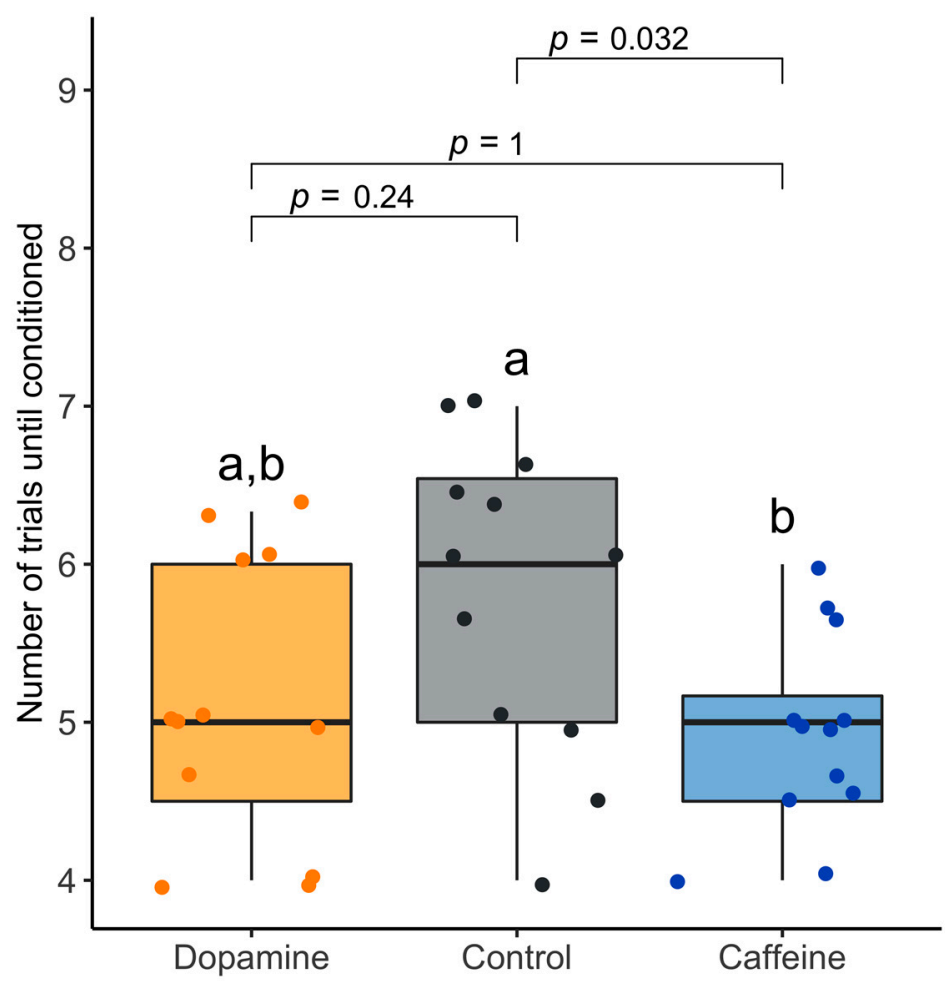

Figure 2. The effect of caffeine and dopamine on learning performance in a European honeybee colony, Apis mellifera. Caffeine-treated bees showed a higher learning performance than the control, requiring less training trials until conditioned to the stimulus. Significance was calculated using repeated measures ANOVA and differences between groups were estimated using Multiple Comparisons of Means (Tukey Contrasts). 


\subsection{Classroom Discussion}

Outreach projects are an essential tool for communicating and disseminating science to non-specific audiences. Although they usually lack a common structure, outreach activities are better internalised by the public when they are involved in major stages of data collection and discussion. As such, our practice gathered all major elements present in the scientific method: (a) the research subject was presented in a logical, sequential form using slides; (b) based on background information provided, participants were instructed to build scientific hypothesis, study aims and predictions; (c) data collection was performed based on a protocol and individuals were given full autonomy on their research; (d) statistical analysis was conducted as group based on the results provided; (e) all participants engaged in a collective discussion aiming to understand biological mechanisms that might have influenced the results; (f) new hypotheses and developments were suggested that could be implemented in a future event.

\section{Discussion}

\subsection{Bee Cognition}

In classroom-based examination of honeybee cognition, we sought to employ a highly recognisable treatment to facilitate student engagement. Caffeine is the most consumed psychoactive in the world, being used by different cultures and social groups to promote wakefulness. Similar to other psychoactive drugs, caffeine also affects dopamine signalling, by blocking dopamine transporters, stimulating its release from terminals and reducing reuptake [44-46]. Dopamine is a neurotransmitter with multiple functions, such as the control of reward-motivated behaviour. Therefore, dopamine is an essential component in conditioning, memory and learning [47-49], being present in most multicellular animals [50]. The group-generated data identified caffeine-induced improvements in honeybee learning when compared to dopamine supplementation or no supplementation control. This was potentially due to caffeine increasing the dopamine production and reducing reuptake [44-46]. As a result, caffeine treated bees potentially had more available dopamine than dopamine treated bees.

It is important to note that this engagement protocol sacrifices data robustness for safety and reproducibility. For example, newly emerged bees have a greatly diminished aptitude for conditioning relative to older bees [41], but as stated, lack venom, and are therefore safer for classroom use. In addition, we used honeybees from a single hive. This pseudo replication reduces the cost considerably and facilitates experimental setup as a single honeybee hive is used. In addition, concentrations of caffeine and dopamine much higher than those found naturally were employed [5,51-53]. This facilitated a drug-induced detectable phenotype. However, we acknowledge that these limitations and experimental design choices greatly reduce the generality and reliability of the data. Multiple groups of non-specialist participants can be leveraged to generate large datasets, but these datasets should be viewed more as pilot studies.

\subsection{Insect-Based Engagement}

Insects are now facing an unprecedented threat [54]. Worldwide, their numbers have plummeted, but because of their size, and relative unimportance to the average citizen, scientists do not know the exact extent of their decline [54]. Educating the public by demystifying their presence, function and importance is imperative to solve this crisis $[10,55,56]$. Projects that stimulate contact and promote mutual respect between humans and invertebrates, such as citizen science, are more important today than ever $[10,55,56]$.

Among insects, the European honeybee is an ideal candidate for citizen science studies. They are likable, relatively docile (when carefully handled) and, because of their biology, they can have direct parallels with humans. For instance, they live in a society, they share food, communicate locations and even the necessity of grooming through the grooming dance [57]. Furthermore, their relatively larger brain, compared to another laboratory 
staple, the fruit fly Drosophila melanogaster, makes learning experiments simpler and easier to conduct with non-specialists $[20,24]$.

We propose using honeybees to highlight the importance of insects to global ecology and economic prosperity. Here we describe a straightforward and engaging activity that can be widely deployed to facilitate this. We do, however, appreciate that the sourcing and handling of bees is a potential issue in conducting this experiment. A potential solution to this is partnership with a local apiarist or an apiarist society.

\subsection{Data Quality and Loss}

The 20 percent loss in data observed in our experiment was well within what we consider acceptable given the complex experimental system employed. This loss is compensated by the increase in data points a project like this achieves. The experiment was conducted on a relatively small scale, and thus, a limited number of samples was provided per experimental group (three bees per experimental group). Given the simplicity and low cost related to the experimental setup, a larger number of replicates could have been provided to the Ryukyu Girls group to compensate for any data loss.

\subsection{Absence of Adverse Events}

No adverse events, in particular bee stings, occurred during this activity. Honeybees pose an additional risk when compared to other model systems but when effectively managed this risk is greatly minimised. However, it is paramount that any honeybee-based engagement activities are mindful of the risks posed and take steps to mitigate these risks. Although strong advocates for the inclusion of honeybees in activities involving individuals untrained in their handling, we also recognise the need for restraining the bees.

\subsection{Extension to Other Teaching Scenarios}

We propose that these methods could also be employed in classroom-based activities. With limited modification, our protocol could be extended to include teaching on hypothesis development and the scientific method generally. Moreover, lessons on data analysis, specifically in $\mathrm{R}$, could also be included using markdown code we have shared. Finally, the additional training undergraduate students undertake throughout their education may also facilitate the more robust experimental designs discussed above without compromising safety.

\section{Conclusions}

With the ongoing worldwide concerns regarding the collapse of honeybee colonies [58], we hope the use of A. mellifera in outreach, citizen science and education raises awareness and is instrumental in communities adopting more bee-friendly policies. We also hope that through engagement with sections of the community typically absent from scientific discourse, we can amplify this awareness and also foster lifelong critical learning.

Supplementary Materials: The following are available online at https:/ / www.mdpi.com/article/10 .3390/insects12090842/s1, Table S1: Number of trainings trails until individual honeybees associated the lemon scent with a sugary reward, File S1: A copy of the laboratory notebook.

Author Contributions: M.V. designed and developed the honeybee experimental framework with input from all authors. J.A.D., I.K., M.T. and M.V. conducted the engagement activity. All authors wrote and edited the paper. All authors have read and agreed to the published version of the manuscript.

Funding: J.A.D.: I.K. and M.V. were supported by the Okinawa Institute of Science \& Technology. Experimental materials were supported by the Okinawa Institute of Science \& Technology and MV. Subsequent analysis and manuscript collation was supported by JSPS KAKENHI Grants 19K06795 and 19K16205 awarded to J.A.D. and M.V. respectively. The Ryukyu Girls program and participants were supported by Okinawa Institute of Science \& Technology Graduate University, University of the Ryukyus, Okinawan Prefectural Board of Education, Okinawan Prefectural Government, 
the Okinawa Institute of Science and Technology Graduate University Promotion Council and the 'FY2018 GST Female Jr. high and high school student support program.

Institutional Review Board Statement: Not applicable.

Informed Consent Statement: Not applicable.

Data Availability Statement: All data and data analysis present in this manuscript can be viewed at https://github.com/marivelasque/HoneybeeOutreach.git.

Acknowledgments: We would like to acknowledge the support of the Okinawa Institute of Science and Technology Diversity Section. We would also like to thank all participants of the 2018 Ryukyu Girls program for their enthusiasm and dedication.

Conflicts of Interest: The authors declare no conflict of interest.

\section{References}

1. Cook, J. Countering Climate Science Denial and Communicating Scientific Consensus; Oxford University Press: Oxford, UK, 2016.

2. Howarth, C.; Parsons, L.; Thew, H. Effectively communicating climate science beyond academia: Harnessing the heterogeneity of climate knowledge. One Earth 2020, 2, 320-324. [CrossRef]

3. Klenert, D.; Funke, F.; Mattauch, L.; O'Callaghan, B. Five lessons from COVID-19 for advancing climate change mitigation. Environ. Resour. Econ. 2020, 76, 1-28. [CrossRef]

4. Decourtye, A.; Lacassie, E.; Pham-Delègue, M.-H. Learning performances of honeybees (Apis mellifera $\mathrm{L}$ ) are differentially affected by imidacloprid according to the season. Pest Manag. Sci. 2003, 59, 269-278. [CrossRef]

5. Si, A.; Zhang, S.-W.; Maleszka, R. Effects of caffeine on olfactory and visual learning in the honey bee (Apis mellifera). Pharmacol. Biochem. Behav. 2005, 82, 664-672. [CrossRef]

6. Varnon, C.A.; Dinges, C.W.; Vest, A.J.; Abramson, C.I. Conspecific and interspecific stimuli reduce initial performance in an aversive learning task in honey bees (Apis mellifera). PLoS ONE 2020, 15, e0228161. [CrossRef]

7. Herodotou, C.; Aristeidou, M.; Sharples, M.; Scanlon, E. Designing citizen science tools for learning: Lessons learnt from the iterative development of nQuire. Res. Pract. Technol. Enhanc. Learn. 2018, 13, 4. [CrossRef]

8. Ferguson, R.; Jones, A.; Scanlon, E. Educational Visions: The Lessons from 40 Years of Innovation; Ubiquity Press: London, UK, 2019.

9. Ramos, D.L.; Cunha, W.L.; Evangelista, J.; Lira, L.A.; Rocha, M.V.C.; Gomes, P.A.; Frizzas, M.R.; Togni, P.H.B. Ecosystem services provided by insects in Brazil: What do we really know? Neotrop. Entomol. 2020, 49, 783-794. [CrossRef] [PubMed]

10. Del-Claro, K.; Stefani, V.; Lange, D.; Vilela, A.A.; Nahas, L.; Velasques, M.; Torezan-Silingardi, H.M. The importance of natural history studies for a better comprehension of animal-plant interaction networks. Biosci. J. 2013, 439-448. [CrossRef]

11. Losey, J.E.; Vaughan, M. The economic value of ecological services provided by insects. Bioscience 2006, 56, 311. [CrossRef]

12. Del-Claro, K.; Torezan-Silingardi, H.M. Insect-plant interactions: New pathways to a better comprehension of ecological communities in Neotropical savannas. Neotrop. Entomol. 2009, 38, 159-164. [CrossRef] [PubMed]

13. Del-Claro, K. Multitrophic relationships, conditional mutualisms, and the study of interaction biodiversity in tropical savannas. Neotrop. Entomol. 2004, 33. [CrossRef]

14. Oliveira, J.B.B.S.; Faria, M.L.; Borges, M.A.Z.; Fagundes, M.; Araújo, W.S. Comparing the plant-herbivore network topology of different insect guilds in Neotropical savannas. Ecol. Entomol. 2020, 45, 406-415. [CrossRef]

15. Vilela, A.A.; Del Claro, V.T.S.; Torezan-Silingardi, H.M.; Del-Claro, K. Climate changes affecting biotic interactions, phenology, and reproductive success in a savanna community over a 10-year period. Arthropod Plant Interact. 2018, 12, 215-227. [CrossRef]

16. Wolda, H. Altitude, habitat and tropical insect diversity. Biol. J. Linn. Soc. Lond. 1987, 30, 313-323. [CrossRef]

17. Kiatoko, N.; Raina, S.K.; van Langevelde, F. Impact of habitat degradation on species diversity and nest abundance of five African stingless bee species in a tropical rainforest of Kenya. Int. J. Trop. Insect Sci. 2017, 37, 189-197. [CrossRef]

18. Cuartas-Hernández, S.E.; Gómez-Murillo, L. Effect of biotic and abiotic factors on diversity patterns of anthophyllous insect communities in a tropical mountain forest. Neotrop. Entomol. 2015, 44, 214-223. [CrossRef]

19. Babin-Fenske, J.; Anand, M. Terrestrial insect communities and the restoration of an industrially perturbed landscape: Assessing success and surrogacy. Restor. Ecol. 2010, 18, 73-84. [CrossRef]

20. Menzel, R. A short history of studies on intelligence and brain in honeybees. Apidologie 2021, 52, 23-34. [CrossRef]

21. I'Anson Price, R.; Grüter, C. Why, when and where did honey bee dance communication evolve? Front. Ecol. Evol. 2015, 3. [CrossRef]

22. Avarguès-Weber, A.; Giurfa, M. Conceptual learning by miniature brains. Proc. Biol. Sci. 2013, 280, 20131907. [CrossRef] [PubMed]

23. Avarguès-Weber, A.; Dyer, A.G.; Giurfa, M. Conceptualization of above and below relationships by an insect. Proc. Biol. Sci. 2011, 278, 898-905. [CrossRef]

24. Menzel, R. The honeybee as a model for understanding the basis of cognition. Nat. Rev. Neurosci. 2012, 13, 758-768. [CrossRef]

25. Pavlov, P.I. Conditioned reflexes: An investigation of the physiological activity of the cerebral cortex. Ann. Neurosci. 2010, 17, 136-141. [CrossRef]

26. Braun, G.; Bicker, G. Habituation of an appetitive reflex in the honeybee. J. Neurophysiol. 1992, 67, 588-598. [CrossRef] [PubMed] 
27. Gil, M.; De Marco, R.J. Olfactory learning by means of trophallaxis in Apis mellifera. J. Exp. Biol. 2005, 208, 671-680. [CrossRef] [PubMed]

28. Smith, B.H.; Burden, C.M. A proboscis extension response protocol for investigating behavioral plasticity in insects: Application to basic, biomedical, and agricultural research. J. Vis. Exp. 2014, e51057. [CrossRef] [PubMed]

29. Frost, E.H. Using the Proboscis Extension Reflex to Evaluate Effects of Stressors on Honeybee Learning and Memory; Acadia University: Wolfville, NS, Canada, 2011.

30. Pratavieira, M.; da Silva Menegasso, A.R.; Roat, T.; Malaspina, O.; Palma, M.S. In situ metabolomics of the honeybee brain: The metabolism of l-arginine through the polyamine pathway in the proboscis extension response (PER). J. Proteome Res. 2020, 19, 832-844. [CrossRef] [PubMed]

31. Hostachy, C.; Couzi, P.; Hanafi-Portier, M.; Portemer, G.; Halleguen, A.; Murmu, M.; Deisig, N.; Dacher, M. Responsiveness to sugar solutions in the moth Agrotis ipsilon: Parameters affecting proboscis extension. Front. Physiol. 2019, 10, 1423. [CrossRef]

32. Giurfa, M.; Sandoz, J.-C. Invertebrate learning and memory: Fifty years of olfactory conditioning of the proboscis extension response in honeybees. Learn. Mem. 2012, 19, 54-66. [CrossRef]

33. Giurfa, M. Behavioral and neural analysis of associative learning in the honeybee: A taste from the magic well. J. Comp. Physiol. Neuroethol. Sens. Neural Behav. Physiol. 2007, 193, 801-824. [CrossRef]

34. Bennett, M.M.; Cook, C.N.; Smith, B.H.; Lei, H. Early olfactory, but not gustatory processing, is affected by the selection of heritable cognitive phenotypes in honey bee. J. Comp. Physiol. Neuroethol. Sens. Neural Behav. Physiol. 2021, 207, 17-26. [CrossRef]

35. Hori, S.; Takeuchi, H.; Arikawa, K.; Kinoshita, M.; Ichikawa, N.; Sasaki, M.; Kubo, T. Associative visual learning, color discrimination, and chromatic adaptation in the harnessed honeybee Apis mellifera L. J. Comp. Physiol. Neuroethol. Sens. Neural Behav. Physiol. 2006, 192, 691-700. [CrossRef]

36. Niggebrügge, C.; Leboulle, G.; Menzel, R.; Komischke, B.; de Ibarra, N.H. Fast learning but coarse discrimination of colours in restrained honeybees. J. Exp. Biol. 2009, 212, 1344-1350. [CrossRef] [PubMed]

37. Scheiner, R.; Abramson, C.I.; Brodschneider, R.; Crailsheim, K.; Farina, W.M.; Fuchs, S.; Grünewald, B.; Hahshold, S.; Karrer, M.; Koeniger, G.; et al. Standard methods for behavioural studies ofApis mellifera. J. Apic. Res. 2013, 52, 1-58. [CrossRef]

38. Poddar, R.; Kawai, R.; Ölveczky, B.P. A fully automated high-throughput training system for rodents. PLoS ONE 2013,8 , e83171. [CrossRef]

39. Kirkerud, N.H.; Wehmann, H.-N.; Galizia, C.G.; Gustav, D. APIS-a novel approach for conditioning honey bees. Front. Behav. Neurosci. 2013, 7, 29. [CrossRef]

40. Hladun, K.R.; Smith, B.H.; Mustard, J.A.; Morton, R.R.; Trumble, J.T. Selenium toxicity to honey bee (Apis mellifera L.) pollinators: Effects on behaviors and survival. PLOS ONE 2012, 7, e34137. [CrossRef]

41. Behrends, A.; Scheiner, R. Evidence for associative learning in newly emerged honey bees (Apis mellifera). Anim. Cogn. 2009, 12, 249-255. [CrossRef] [PubMed]

42. Owen, M.D.; Braidwood, J.L. A quantitative and temporal study of histamine and histidine in honey bee (Apis mellifera L.) venom. Can. J. Zool. 1974, 52, 387-392. [CrossRef] [PubMed]

43. Elias-Neto, M.; Nascimento, A.L.O.; Bonetti, A.M.; Nascimento, F.S.; Mateus, S.; Garófalo, C.A.; Bitondi, M.M.G. Heterochrony of cuticular differentiation in eusocial corbiculate bees. Apidologie 2014, 45, 397-408. [CrossRef]

44. Nehlig, A.; Daval, J.L.; Debry, G. Caffeine and the central nervous system: Mechanisms of action, biochemical, metabolic and psychostimulant effects. Brain Res. Brain Res. Rev. 1992, 17, 139-170. [CrossRef]

45. Nehlig, A. (Ed.) Coffee, Tea, Chocolate, and the Brain; Nutrition, Brain and Behavior; CRC Press: London, UK, 2010; ISBN 9780415306911.

46. Volkow, N.D.; Wang, G.-J.; Logan, J.; Alexoff, D.; Fowler, J.S.; Thanos, P.K.; Wong, C.; Casado, V.; Ferre, S.; Tomasi, D. Caffeine increases striatal dopamine D2/D3 receptor availability in the human brain. Transl. Psychiatry 2015, 5, e549. [CrossRef] [PubMed]

47. Fadok, J.P.; Dickerson, T.M.K.; Palmiter, R.D. Dopamine is necessary for cue-dependent fear conditioning. J. Neurosci. 2009, 29, 11089-11097. [CrossRef] [PubMed]

48. Schultz, W. Neuronal reward and decision signals: From theories to data. Physiol. Rev. 2015, 95, 853-951. [CrossRef]

49. Waddell, S. Reinforcement signalling in Drosophila; dopamine does it all after all. Curr. Opin. Neurobiol. 2013, 23, 324-329. [CrossRef]

50. Barron, A.B.; Søvik, E.; Cornish, J.L. The roles of dopamine and related compounds in reward-seeking behavior across animal phyla. Front. Behav. Neurosci. 2010, 4, 163. [CrossRef]

51. Wright, G.A. The role of dopamine and serotonin in conditioned food aversion learning in the honeybee. Commun. Integr. Biol. 2011, 4, 318-320. [CrossRef]

52. Wright, G.A.; Baker, D.D.; Palmer, M.J.; Stabler, D.; Mustard, J.A.; Power, E.F.; Borland, A.M.; Stevenson, P.C. Caffeine in floral nectar enhances a pollinator's memory of reward. Science 2013, 339, 1202-1204. [CrossRef]

53. Thomson, J.D.; Draguleasa, M.A.; Tan, M.G. Flowers with caffeinated nectar receive more pollination. Arthropod Plant Interact. 2015, 9, 1-7. [CrossRef]

54. Goulson, D. The insect apocalypse, and why it matters. Curr. Biol. 2019, 29, R967-R971. [CrossRef]

55. Saunders, M.E.; Janes, J.K.; O'Hanlon, J.C. Moving on from the insect apocalypse narrative: Engaging with evidence-based insect conservation. Bioscience 2020, 70, 80-89. [CrossRef] 
56. Samways, M.J. The need for insect diversity conservation. In Insect Diversity Conservation; Cambridge University Press: Cambridge, UK, 2010; pp. 1-2, ISBN 9780511614163.

57. Land, B.B.; Seeley, T.D. The grooming invitation dance of the honey bee. Ethology 2004, 110, 1-10. [CrossRef]

58. Roy, D.; Debnath, P.; Mondal, D.; Sarkar, P. Colony collapse disorder of honey bee: A neoteric ruction in global apiculture. Curr. J. Appl. Sci. Technol. 2018, 26,1-12. [CrossRef] 